\title{
Comparisons of adaptive median filter based on homogeneity level information and the new generation filters
}

\author{
Mr Praveen Kumar B.T ${ }^{1}$,Dr. R. Vijayakumar ${ }^{2}$ \\ Research scholar, MahatmaGandhi university, kottayam,kerala,India \\ Director ,School of computer science, MahathmaGandhi university, kottayam,kerala,India
}

\begin{abstract}
This paper deals with the comparisons of various filters such as fuzzy based filters and discrete wavelet based filters and continuous wavelet based filters, and adaptive median filters based on homogeneity level information. One of the difficult tasks in the image processing is the removal of impulse noise such as random valued impulse noise and salt and pepper noise. Different type of noise removal filters can be implemented based on current technology. In this paper the comparison of the filter efficiency can be done by the help of the factors such as MSE,PSNR. The result comparisons of different filters are implemented by the help of MATHLAB
\end{abstract}

Keywords: Adaptive median filter, continuous wavelet filter. discrete wavelet filters, fuzzy based filter, salt and pepper noise,

\section{Introduction}

One of the major areas in the image processing is the image denoising. The noise can be formed in the image due to some factors affecting the image abstraction and transmission, and the storage time. That is the imperfect Instruments used in the image processing time.There are different filters developed for the image denoising based on different conditions. In the median filter the noise can be removed by pixel based and the adaptive type filter the noise can be removed by first detect the noisy pixel and remove the noise by filtering. In fuzzy based filtering is only apply on the corrupted pixels in the image while uncorrupted pixels are left unchanged. Wavelet analysis is a new development in the area of applied mathematics. Wavelet transforms have become well known as useful tools for various signal processing applications. Wavelets are mathematical functions that allow complex information to be decomposed into different frequency components, and then study each component with a resolution matched to its scale. Wavelet means small waves. The wavelets can be built by taking a different shape, called a mother wavelet, and dilating, compressing or shifting it in time. The wavelet are classified as continuous wavelet transforms (CWTs) and discrete wavelet transforms (DWTs).The continuous wavelet transform is best suited to signal analysis In the filtering process the main problem in bluring.Blurring is the form of bandwidth reduction of images caused by imperfect image formation process such as relative motion between camera and original scene or by an optical system that is out of focus .

Its semi discrete version and its fully discrete one (the discrete wavelet transform) have been used for signal coding applications, including image compression, image filtering and various tasks in computer vision .

\section{Impulse Noise Models}

Noise can be classified as salt-and-pepper noise (SPN) and random-valued impulse noise (RVIN). Let us consider an image with pixel value the noise can be classified as

$$
P(i j)=\left\{\begin{array}{c}
Q(i j) \text { with probability } P \\
X(i j) \text { without probability } 1-P
\end{array}\right.
$$

That is the image $P(i, j)$ is the sum of $X(i, j)$ and $Q(i, j)$.Let $x(i, j)$ denote the noisy pixel and $Q(i, j)$ denote the noise free pixel. In salt and pepper noise noisy pixels take either minimal or maximal values. That is in gray level image the pixel value is only 0 or 255 and for random-valued impulse noise, noisy pixels take any value within the range minimal to maximal value. That is in gray level image the pixel value is lies between 0 and 255

\section{Adaptive Median Filter Based On Homogenity Level Information.}

Adaptive median filter based on homogeneity level information is a. decision-based, signal adaptive median filtering algorithm for removal of impulse noise. In this algorithm achieves accurate noise detection and high SNR measures without smearing the fine details and edges in the image. The notion of homogeneity level is defined for pixel values based on their global and local statistical properties. The co occurrence matrix technique is used to represent the correlations between a pixel and its neighbors, and to derive the upper and lower bound of the homogeneity level. Noise detection is performed at two stages: noise candidates are first selected using the homogeneity level, and then a refining process follows to eliminate false detections. The 
noise detection scheme does not use a quantitative decision measure, but uses qualitative structural information, and it is not subject to burdensome computations for optimization of the threshold values. The two major functions in this filter Is decisions making and noise filtering. First detects the noise on based of homogeneity of pixel and then apply the filter for the noise pixel. The major step in the filtering algorithm is calculate the bounds of the homogeneity level then detection of impulse noise and refined the selection of impulse noise and the final step apply the median filter for the noisy pixel The fig(1) represents different steps for denoising the lena image of $20 \%$ salt and pepper noise.

\section{Wavelet Based Median Filters.}

Basically the wavelet based filters are two types one discrete wavelet transform and another is continuous wavelet transform. The wavelet analysis described as the continuous wavelet transform or $C W T$. More formally it is written as

$$
\gamma(s, \tau)=\int f(t) \Psi_{s, \tau}^{*}(t) d t,
$$

where * denotes complex conjugation. This equation shows how a function $f(t)$ is decomposed into a set of basis functions $\Psi_{s, \tau}^{*}(t)$ called the wavelets. The variables $s$ and $\tau$ are the new dimensions, scale and translation, after the wavelet transform. For completeness sake equation(3)give the inverse wavelet transform

$$
f(t)=\iint \gamma(s, \tau) \Psi_{s, \tau}(t) d \tau d s .
$$

The wavelets are generated from a single basic wavelet $\Psi(t)$ the so-called mother wavelet, by scaling and translation:

$$
\Psi_{s, \tau}=\frac{1}{\sqrt{\mathrm{s}}} \Psi\left(\frac{\mathrm{t}-\tau}{\mathrm{s}}\right)
$$

In equation (4) $s$ is the scale factor $\tau$ is the translation factor

Discrete wavelets are not continuously scalable and translatable but can only be scaled and translated in discrete steps. This is achieved by modifying the wavelet representation (5) to create

$$
\Psi_{j, k}(\mathrm{t})=\frac{1}{\sqrt{s_{o}^{j}}} \Psi\left(\frac{\mathrm{t}-\mathrm{k} \tau_{0} \mathrm{~s}_{0}^{\mathrm{j}}}{\mathrm{s}_{\mathrm{o}}^{\mathrm{j}}}\right)
$$

\section{Discreate Wavelet Based And Continious Wavelet Based Filters}

The Discrete wavelet based image filter architecture includes transforms modules, a RAM and bus interfaces. This architecture works in non separable fashion using a serial-parallel filter with distributed control to compute all the DWT (1D-DWT and 2D-DWT) resolution levels. The fig(2) represents the one level of 2DDWT. The so-called lifting scheme represents the fastest implementation of the DWT. A VHDL model was described and synthesized using implementation of architecture. Discrete Wavelet Transform (DWT) based image coding has better performance than traditional DCT based image coding, especially for low bit-rate applications. Therefore many famous coders have been proposed to effectively compress images or frames processed via DWT.In the wavelet transformations thresholding function is one of the major factor. The thresholding.function can be classified in to two types that is soft thresholding and hard thresholding. The fig(3) represents different steps for denoising the lena image of $20 \%$ salt and pepper noise.

In continuous wavelet (CWT) based noise filter also used the mother wavelet such as Haar Daubechies. Wavelet based filter are very much power full than the other type of filters and this type used for climate analysis and compression, medical fields etc. The fig(4) represents different steps for denoising the of filter are lena image of $20 \%$ salt and pepper noise

\section{Fuzzy Logic Based Adaptive Noise Filters}

Fuzzy logic based Adaptive Noise filter for real time image processing applications. Initially, the detection is performed using $3 \times 3$ scan and then taking a mean of four pixels further scanning is performed and then applied the fuzzy rules. For improving the image quality, textures and edges the histogram approach is applied. The two steps are first detection stage will identify the noise pixels, the second stage is filtering if pixels are noise-free then they are left unprocessed. This filter uses fuzzy reasoning to remove uncertainty present in the information as introduced by the noise. The conventional median filter is applied for removing salt and pepper noise .To remove salt \&pepper noise is by windowing the noisy image that will affecting the process of filtering exhibits blurring of filtered images. The fig(5) represents different steps for denoising the lena image of $20 \%$ salt and pepper noise. 


\section{Figures And Tables}
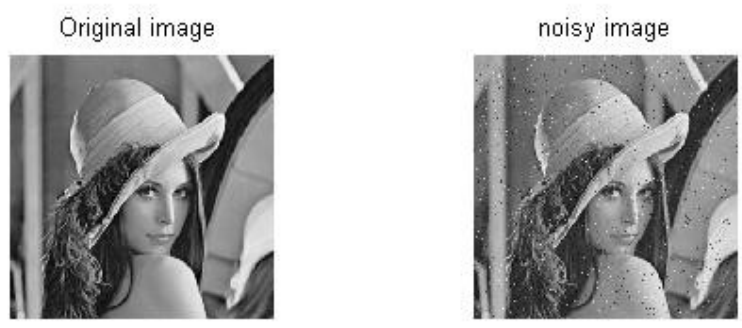

$$
\text { false detection }
$$

Adaptive denoised image

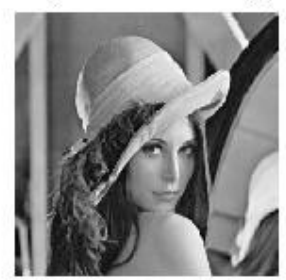

Fig(1).Noise identification and filtering of $20 \%$ salt and pepper added Lena image.

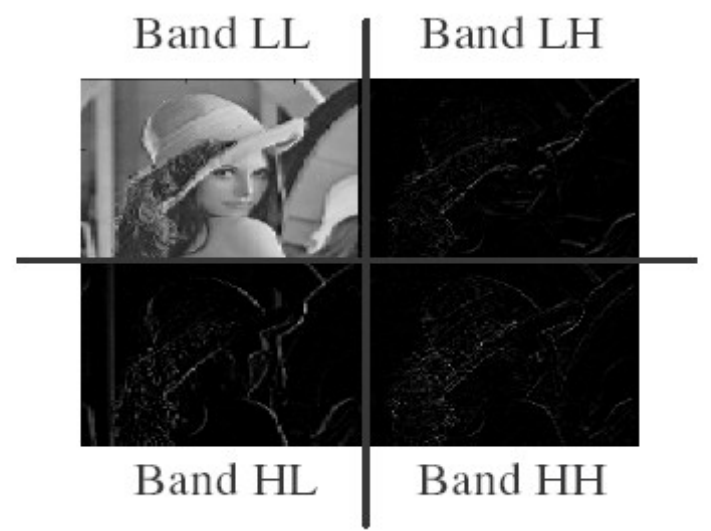

Fig(2) One level of 2D -DWT
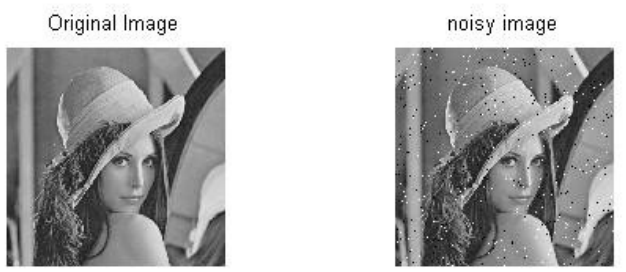

$$
\text { denoise image }
$$

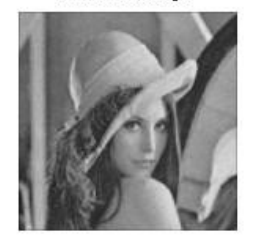

Fig(3).Noise identification and filtering of $20 \%$ salt and pepper added image using DWT 

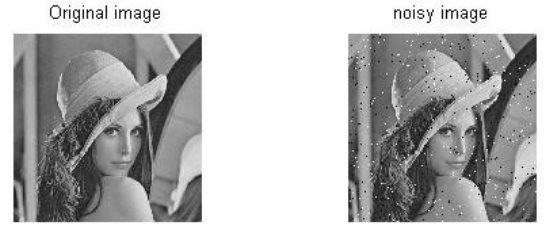

De-noised image

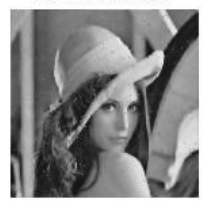

Fig(4).Noise identification and filtering of 20\% salt and pepper added image using CWT
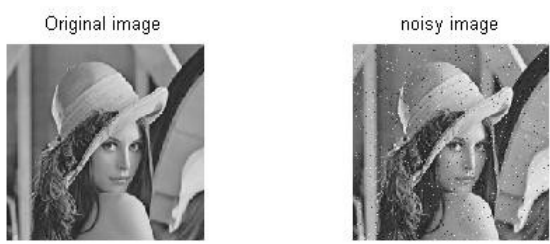

denoised image

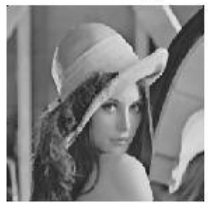

Fig(5).Noise identification and filtering of $20 \%$ salt and pepper added image using fuzzy filter. Performance comparison results for Lena Image of $20 \%$ noise.

\begin{tabular}{|l|l|l|l|}
\hline Type of filter & MSE & PSNR & RMSE \\
\hline Adaptive median filter & 29.48 & 33.44 & 5.43 \\
\hline Discrete wavelet & 502.41 & 21.12 & 22.41 \\
\hline Continuous wavelet & 411.27 & 21.99 & 20.28 \\
\hline Fuzzy based filter & 2.56 & 44.05 & 1.06 \\
\hline
\end{tabular}

Performance comparison results for cameraman Image of $20 \%$ noise.

\begin{tabular}{|l|l|l|l|}
\hline Type of filter & MSE & PSNR & RMSE \\
\hline Adaptive median filter & 206.33 & 31.99 & 14.36 \\
\hline Discrete wavelet & 960.86 & 20.12 & 31.00 \\
\hline Continuous wavelet & 580.69 & 21.49 & 24.10 \\
\hline Fuzzy based filter & 4.00 & 40.01 & 2.00
\end{tabular}

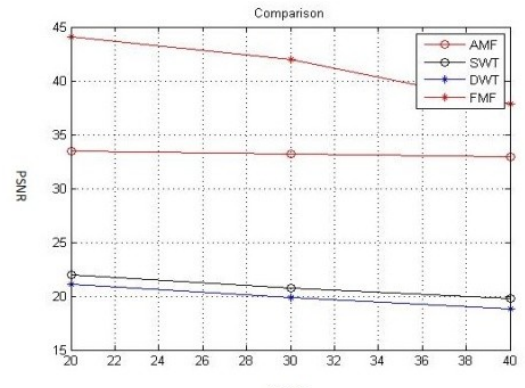

Performance comparison of different filtering methods on image degraded by salt and pepper impulse noise 


\section{Conclusion}

This paper mainly deals with the comparisons of adaptive median filter based on homogeneity level information, discrete wavelet based filer, continuous wavelet based filter and fuzzy based noise filter for the removal of salt and pepper noise removal. The fuzzy based filter performs the highest PSNR rate rather than the other type of filters. The result can be simulated using MATHLAB2010.

\section{Acknowlegment}

To complete this work, I have got valuable suggestions and guidance from different experts of this field. The Book Digital Image Processing (Third Edition) by Rafael C. Gonzalwz and Richard E. Woods is very helpful for beginners' to start research in this field .

\section{References}

[1] J. Astola and P. Kuosmanen, Fundamentals of Nonlinear Digital Filtering. Boca Raton, CRC, 1997.

[2] T. Chen and H. R. Wu, "Space variant median filters for the restoration of impulse noise corrupted images," IEEE Transactions on Circuits and Systems II, 48 (2001), pp. 784-789.

[3] R. C. Gonzalez and R. E. Woods, Digital Image Processing Second Edition, Prentice Hall, 2001; and Book Errata Sheet (July 31, 2003), http://www.imageprocessingbook.com/downloads/errata sheet.htm.

[4] K.P. Soman and K.I. Ramachandran, "Insight into Wavelets from Theory to Practice".

[5] K. K. V. Toh, H. Ibrahim, and M. N. Mahyuddin,"Salt-and-pepper noise detection and reduction using fuzzy switching median filter," IEEE Trans. ConsumerElectron., vol. 54, no. 4, pp. 1956-1961, Nov. 2008.

[6] Farzam Farbiz, Mohammad Bager Menhaj, Seyed A. Motamedi, and Martin T. Hagan, "A new Fuzzy Logic Filter for image Enhancement” IEEE Transactions on Systems, Man, And Cybernetics-Part B: Cybernetics, Vol. 30, No. 1, February 2000

[7] Robert D. Nowak, "Wavelet Based Rician Noise Removal", IEEE Transactions on Image Processing,vol. 8, no. 10, pp.1408, October 1999.

[8] Geoffrine Judith.M.C1 and N.Kumarasabapathy, "STUDY AND ANALYSIS OF IMPULSE NOISE REDUCTION FILTERS”, Signal \& Image Processing : An International Journal(SIPIJ) Vol.2, No.1, pp. 82-92,March 2011 